\title{
Function and regulation of an aldehyde dehydrogenase essential for ethanol and methanol metabolism of the yeast, Komagataella phaffii
}

\author{
Running title: Function and regulation of $K$. phaffii aldehyde dehydrogenase
}

\section{Kamisetty Krishna Rao ${ }^{1}$, Umakant Sahu ${ }^{1, \#}$ and Pundi N Rangarajan ${ }^{1, *}$}

${ }^{1}$ Department of Biochemistry, Indian Institute of Science, Bangalore 560012, INDIA

"Present address: Department of Biochemistry and Molecular Genetics, Northwestern University Feinberg School of Medicine, Chicago, IL 60611

"To whom correspondence should be addressed: Pundi Rangarajan, Department of Biochemistry, Indian Institute of Science, Bangalore 560012, INDIA. Email: chair.bc@,iisc.ac.in, Tel: 918022932213

Key words: aldehyde dehydrogenase, Mxr1p, transcriptional regulation, alcohol oxidase, yeast metabolism

\begin{abstract}
The genome of the methylotrophic yeast, Komagataella phaffii harbours multiple genes encoding putative alcohol dehydrogenases and aldehyde dehydrogenases (ALDs). Here, we demonstrate that one of the ALDs denoted as ALD-A is essential for ethanol metabolism. A zinc finger transcription factor known as Mxrlp regulates $A L D-A$ transcription by binding to Mxr1p response elements (MXREs) in the $A L D-A$ promoter. Mutations which abrogate Mxrlp binding to $A L D-A$ MXREs in vitro abolish transcriptional activation from $A L D-A$ promoter in vivo. Mxr1p regulates $A L D-A$ expression during ethanol as well as methanol metabolism. ALD-A is essential for the utilization of methanol and $\Delta$ ald- $a$ is deficient in alcohol oxidase (AOX), a key enzyme of methanol metabolism. AOX protein but not mRNA levels are down regulated in $\Delta$ ald-a. ALD-A and AOX localize to cytosol and peroxisomes respectively during methanol metabolism suggesting that they are unlikely interact with each other in vivo. This study has led to the identification of Mxrlp as a key regulator of $A L D-A$ transcription during ethanol and methanol metabolism of $K$. phaffii. Post-transcriptional regulation of AOX protein levels by ALD-A during methanol metabolism is another unique feature of this study.
\end{abstract}

\section{INTRODUCTION}

In $S$. cerevisiae, ethanol is metabolized into acetaldehyde, acetate and acetyl-CoA by the activity of alcohol dehydrogenase (ADH), aldehyde dehydrogenase (ALD) and acetyl-CoA synthetase (ACS) respectively. These enzymes are encoded by multiple genes which are well characterized. For example, ADH1 functions as the major fermentative enzyme during glucose metabolism, catalyzing the conversion of acetaldehyde to ethanol $(1,2)$. $\mathrm{ADH} 2$ is the major enzyme in the utilization of ethanol as carbon source its expression is repressed by glucose (3-6). Both ADH1 and ADH2 are cytosolic enzymes. A third isozyme, $\mathrm{ADH} 3$, localized in the mitochondria, is a component of the ethanol/acetaldehyde shuttle (7). ADH4 is most closely related to a bacterial, iron-activated alcohol dehydrogenase but requires zinc for its activity like the other $S$. cerevisiae ADH proteins (8-10). ADH5 codes for a minor activity and has not been well characterized (11).

Aldehyde dehydrogenases (ALDs) play a key role during growth of yeasts on nonfermentable carbon sources by converting acetaldehyde generated from ethanol to acetate. They are also involved in the breakdown of toxic aldehydes accumulated under stress conditions (12). In $S$. cerevisiae, ALDs are encoded by five genes: ALD1/ALD6 (YPL061w), ALD2 (YMR170c) and $A L D 3$ (YMR169c), which encode cytosolic isoforms, as well as ALD4 (YOR374w) and ALD5 (YER073w), which encode 
mitochondrial isoforms (13). Several isoenzymes of $S$. cerevisiae ALD are categorized based on their subcellular locations. ALD2, ALD3 and ALD6 encode cytosolic aldehyde hydrogenases whereas ALD4 and ALD5 encode mitochondrial enzymes (14). Of these, ALD4 and ALD6 are the two major enzymes. Expression of $A L D 6$ and $A L D 4$ is induced and repressed by glucose respectively. Expression of $A L D 4$ can be induced in the presence of ethanol and acetaldehyde (14). The carbon source-responsive zinc finger transcription factors, Adrlp and Cat8p regulate $S$. cerevisiae $A D H 2$ expression by binding to upstream activation sequences 1 and 2 respectively in the $A D H 2$ promoter $(6,15,16)$. Adrlp is also required for the activation of genes involved glucose fermentation (e.g., ALD4 and ALD6), glycerol metabolism (e.g., GUT1 and GUT2), fatty acid utilization (e.g., POX1, FOX2), and peroxisome biogenesis (e.g., PEX1) $(17,18)$.

Komagataella phaffii previously known as Pichia pastoris is a methylotrophic yeast and the enzymes of methanol metabolism are well characterized (19). Methanol is converted to formaldehyde by the enzyme alcohol oxidase which is encoded by two genes, $A O X I$ and $A O X 2$. AOX1 is a methanol inducible enzyme and the $A O X I$ promoter is widely used for production of recombinant proteins (19). Unlike $S$. cerevisiae, $K$. phaffii is a respiratory yeast capable of growing to high cell densities when cultured in minimal media containing glucose or glycerol. K. phaffii can also utilize oleic acid, acetate, sorbitol, ethanol or amino acids as the sole source of carbon. Enzymes of ethanol metabolism as well as the regulatory circuits governing their synthesis are not well characterized in $K$. phaffii. Atleast three $K$. phaffii alcohol dehydrogenases referred to as ADH, ADH2, ADH3 have been reported (20-25). $\mathrm{ADH} 3$ is responsible for $92 \%$ of the total alcohol dehydrogenase enzyme activity when cells are grown on glucose media and it shares $73.6 \%$ and $71.4 \%$ amino acid identity with $S$. cerevisiae $\mathrm{ADH} 2$ and $\mathrm{ADH} 3$, respectively (25). K. phaffii ADH3, annotated as mitochondrial alcohol dehydrogenase is likely to be a cytosolic $\mathrm{ADH}$ as it does not possess a mitochondrial targeting sequence $(22,25)$. K. phaffii ADH3 promoter has been exploited for production of recombinant proteins (26). Deletion of $K$. phaffii ADH2 does not affect the growth of $K$. phaffii on glucose or ethanol containing media (21). Acetyl-CoA, a downstream metabolite of ethanol was shown to repress enzymes of methanol metabolism when cultured in presence of ethanol (27).

$K$. phaffii is cultured in minimal medium containing yeast nitrogen base (YNB) or nutrientrich medium containing yeast extract, peptone (YP) supplemented with different sources of carbon such as glucose, glycerol, methanol, ethanol, acetate, glutamate etc. Several transcription factors such as Mxr1p, Rop1p, Trm1p, Nrg1p, Mit1p, Mig1p and Mig2p (19, 2838) regulate the expression of target genes of specific metabolic pathways of $K$. phaffii. In addition to conventional sources of carbon such as glucose and glycerol, $K$. phaffii can utilize amino acids such as glutamate as the sole source of carbon and hence can be cultured in YNB medium containing $2 \%$ glutamate $(\mathrm{YNB}+\mathrm{Glu})$ or $\mathrm{YP}$ medium in which amino acids derived from peptone serve as the source of carbon (38). Mxr1p regulates the expression of several enzymes required for glutamate utilization in cells cultured in YP or $\mathrm{YNB}+\mathrm{Glu}$ (38). Mxr1p regulates the expression of ACS1 encoding acetyl-CoA synthetase 1 during acetate metabolism (37). The target genes of Mxrlp harbour Mxrlp response elements (MXREs) in their promoters to which Mxr1p binds and activates transcription (2831,37,38). Transcription factors regulating ethanol metabolism of $K$. phaffii have not been identified. In $S$. cerevisiae and Kluyveromyces lactis, expression of $A D H 2$ is regulated by $\mathrm{Adr} 1 \mathrm{p}$ considered as the homologue of Mxr1p (15, 39, 40). This study was initiated to examine the role of Mxrlp in the regulation of ethanol metabolism and identify its target genes. Here, we demonstrate that Mxr1p is essential for ethanol metabolism and unlike $S$. cerevisiae Adr1p, Mxr1p does not regulate the expression of $A D H$ genes of $K$. phaffii. Instead, it regulates the transcription of $A L D-A$ encoding one of the four putative ALDs of $K$. phaffii by binding to MXREs in the $A L D-A$ promoter. Interestingly, Mxrlp regulates $A L D-A$ expression during methanol metabolism as well and the ALD-A has a novel role as regulator of AOX, a key enzyme of methanol metabolism.

\section{RESULTS}

Putative alcohol dehydrogenases (ADHs) and aldehyde dehydrogenases (ALDs) of $K$. phaffii 
$K$. phaffii genome contains multiple genes with varying degrees of homology to $S$. cerevisiae $A D H$ and $A L D$ genes. While these are annotated as $K$. phaffii $A D H$ and $A L D$ s, whether they are functional homologues of $S$. cerevisiae $A D H$ and $A L D \mathrm{~s}$ is not clear. Since the exact function of several $K$. phaffii ADHs and ALDs is not known, they were designated using the alphabets $A, B, C, D$ instead of Arabic numerals (1,2,3,4 etc.,) to distinguish them from $S$. cerevisiae enzymes as indicated in Table 1. K. phaffii strains used in this study are listed in Table 2. K. phaffii strains are cultured in a medium containing $0.17 \%$ yeast nitrogen base (YNB) and $2 \%$ glucose (YNBD), $1 \%$ ethanol (YNBE) or $1 \%$ methanol (YNBM).

\section{Transcriptional regulation of $A L D-A$ during ethanol and methanol metabolism by Mxr1p}

The zinc finger transcription factor Mxrlp has emerged as a key regulator of multiple metabolic pathways of $K$. phaffii. However, its role in the regulation of genes of ethanol metabolism is not known. We therefore examined whether Mxrlp is essential for ethanol metabolism by analyzing the growth of K. phaffii GS115 and $\Delta m x r 1$ strains in YNBE. The results indicate that $\Delta m x r l$ exhibits retarded growth in YNBE (Fig. 1A). Utilization of ethanol as a source of carbon involves its sequential conversion into acetaldehyde, acetate and acetyl-CoA by ADH, ALD and ACS respectively (Fig. 1B). K. phaffii genome contains multiple $A D H$ s and $A L D$ s (Table 1). To examine whether genes are expressed, we examined their mRNA levels by qPCR in cells cultured in YNBD and YNBE. The results indicate that $A D H \mathrm{~s}$ and $A L D$ s are actively transcribed during glucose and/or ethanol metabolism (Fig. 1C).

In $S$. cerevisiae, Adr1p, often considered as the homologue of $K$. phaffii Mxrlp $(15,28)$, regulates the expression of $A D H 2$, which encodes aldehyde dehydrogenase 2, the first enzyme of ethanol metabolism (Fig. 1B) (28). We examined whether Mxrlp is required for the expression of any of the $A D H \mathrm{~s}$ in $K$. phaffii by measuring their mRNA levels by qPCR in GS115 and $\Delta m x r 1$ cultured in YNBE. The results indicate that none of the $A D H$ mRNAs are down regulated in $\triangle m x r 1$ (Fig. 1D). Interestingly, $A D H-B$ and $A D H$ -
$D$ mRNAs are upregulated in $\Delta m x r l$ (Fig. 1D) although the significance of their upregulation is not clear at his juncture. Analysis of mRNA levels of $A L D$ s indicate that $A L D-A$ is downregulated in $\triangle m x r 1$ cultured in YNBE (Fig. 1D). Interestingly, $A L D-A$ mRNA levels are also down regulated in $\triangle m x r l$ cultured in YNBM but not YNBD (Fig. 1E). To understand ALD-A function during ethanol and methanol metabolism, K. phaffii $\Delta$ ald$a$ carrying a deletion of $A L D-A$ was generated (Fig. $1 \mathrm{~F}, \mathrm{G})$ and its growth was examined in cells cultured in YNBE and YNBM. Aald- $a$ exhibited impaired growth when cultured in YNBE as well as YNBM (Fig. 1H) indicating that ALD-A is essential for the metabolism of not only ethanol but also methanol. To further confirm regulation of ALD-A expression by Mxr1p, ALD-A was expressed as a Myc-tagged protein (ALD- ${ }^{\mathrm{Myc}}$ ) from $A L D-A$ promoter and ALD-A protein levels were examined by western blot analysis using antiMyc antibodies. ALD-A ${ }^{\mathrm{Myc}}$ levels in $\Delta m x r l$ were lower than those in GS115 cultured in YNBE and YNBM but not YNBD (Fig. 2A). GFP was expressed from $A L D-A$ promoter and its expression was examined by western blotting using anti-GFP antibodies. The results indicate that GFP levels are significantly lower in $\Delta m x r l$ than GS115 cultured in YNBE and YNBM (Fig. 2B). These results confirm that Mxrlp is a key regulator of $A L D-A$ expression. Immunofluorescence studies indicate that ALD$\mathrm{A}^{\mathrm{Myc}}$ predominantly localizes to the cytoplasm of cells cultured in YNBE and YNBM (Fig. 2C).

\section{Identification of Mxr1p response elements (MXREs) in $A L D-A$ promoter $\left(P_{A L D-A}\right)$}

Mxr1p regulates the expression of target genes by binding to MXREs in their promoters which bear the consensus sequence 5' CYCCNY 3' $(29,37,38)$. Analysis of the nucleotide sequence of $1.0 \mathrm{~kb} A L D-A$ promoter indicated the presence of three putative MXREs designated as MXRE1, MXRE2 and MXRE3 (Fig. 3A). Since point mutations within the 5' CYCCNY 3' motif of AOXI MXREs abrogate Mxrlp binding (29), similar mutations were introduced within the 5 , CYCCNY 3' motif of the putative $A L D-A$ MXREs and these were designated as MXRE1-M, MXRE2-M and MXRE3-M (Fig. 3B). The effect 
of these mutations on the binding of recombinant Mxrlp containing $150 \mathrm{~N}$-terminal amino acids including the zinc finger DNA binding domain $\left(\right.$ Mxr1p $\left.{ }^{\mathrm{N} 150}\right)(38)$ to $A L D-A$ MXREs was examined in an electrophoretic mobility shift assay. The results indicate that $\mathrm{Mxrlp}^{\mathrm{N} 150}$ binds to wild type but not the mutant MXREs (Fig. 3C). We examined the ability of Mxrlp to activate transcription from $A L D-A$ promoter containing wild type and mutant MXREs. The gene encoding GFP was cloned downstream of $1.0 \mathrm{~kb} A L D-A$ promoter containing wild type and mutant MXREs (Fig. 4A), transformed into GS115 and GFP expression was examined in cells cultured in YNBE and YNBM. The results indicate that point mutation in MXRE1, 2 or 3 resulted in only a marginal reduction or no reduction in GFP expression (Fig. 4B). However, GFP expression was drastically reduced when all the three or two MXREs were mutated (Fig. 4B-D). These results are summarized in Fig. 4E.

\section{An essential role for ALD-A during methanol metabolism}

The inability of $\Delta a l d-a$ to grow in YNBM suggested that ALD-A is essential for methanol metabolism as well. To gain further insights, we first examined the protein profile of cell lysates of GS115 and $\Delta a l d-a$ cultured in YNBM. AOX is the most abundant protein in cells metabolizing methanol and the protein band can be readily visualized in SDS polyacrylamide gels stained with Coomassie Brilliant Blue R. A protein band corresponding to the molecular weight of AOX was present at high levels in GS115 but not $\Delta$ ald$a$ cultured in YNBM (Fig. 5A). To confirm AOX down regulation in $\Delta a l d-a$, western blot analysis was carried out with anti-AOX antibodies. The results revealed that $\mathrm{AOX}$ protein levels are lower in $\Delta a l d-a$ than GS115 (Fig. 5B). A decrease in AOX protein levels in $\Delta a l d-a$ was not accompanied by a decrease in $A O X 1 \mathrm{mRNA}$ levels (Fig. 5C) suggesting that ALD-A-mediated decrease in AOX protein levels may involve a post-transcriptional mechanism. GS115- $P_{A O X 1^{-}}$ GFP strain contains $P_{A O X I}-G F P$ expression cassette integrated at the His 4 locus (Table 2) and therefore expresses AOX1 protein from the native $A O X 1$ promoter as well as GFP from the $1.0 \mathrm{~kb}$
AOXI promoter at His4 locus. Lysates were prepared from this strain cultured in YNBM, AOX and GFP protein levels were quantitated by western blot analysis using anti-AOX and antiGFP antibodies respectively. Only AOX but not GFP protein levels were reduced in $\Delta a l d-a$ (Fig. 5D) indicating that only AOX protein but not GFP expressed from $A O X 1$ promoter is subjected to ALD-A mediated down regulation. AOX is known to localize to peroxisomes during methanol metabolism (41) and this was confirmed by immunofluorescence using anti-AOX antibodies in GS115 cultured in YNBM (Fig. 5E). Under similar culture conditions, ALD-A ${ }^{\text {Myc }}$ localizes to cytosol (Fig. 5E). ALD-A localizes to cytosol in cells cultured in YNBE as well (Fig. 2C). Thus, AOX and ALD-A localize to two distinct subcellular compartments and therefore unlikely to interact with each other in vivo.

$P$. pastoris genome encodes four ALDs and we examined the effect of deletion of $A L D-D$ on AOX protein levels as well as growth. The results indicate that in AOX protein level in $\Delta a l d$ $d$ is comparable to that of GS115 (Fig. 5F). Further, deletion of $A L D-D$ had no effect on the growth of cells cultured in YNBM and YNBE (Fig. 5G). These results are summarized in Fig. $5 \mathrm{H}, \mathrm{I}$.

\section{DISCUSSION}

In this study, we demonstrate that K. phaffii Mxr1p binds to the MXREs present in the promoter of $A L D-A$ encoding an aldehyde dehydrogenase and activates its transcription during the metabolism of ethanol and methanol. This is the first report on the trans-activation of a gene essential for ethanol metabolism by Mxr1p. Thus far, studies have only focused on the repression of genes of methanol utilization pathway such as $A O X 1$ during ethanol metabolism. It was suggested that acetyl-CoA synthesized during ethanol metabolism may be utilized for the acetylation of histones or other TFs which may repress the expression of methanolinducible genes (Karaoglan et al., 2016b). In another study, phosphorylation of serine 215 residue of Mxr1p was shown to be involved in the transcriptional repression of $A O X I$ during ethanol metabolism (Parua et al., 2012). However, the ability of Mxrlp to activate the expression of gene(s) essential for ethanol metabolism has not 
been investigated. Here, we demonstrate that Mxrlp activates $A L D-A$ transcription during the metabolism of not only ethanol but also methanol. $A L D-A$ is a key target of Mxr1p in cells cultured in YNBE and YNBM. $A L D-A$ promoter $(-1.0 \mathrm{~kb})$ harbours three MXREs to which Mxrlp binds in vitro and activates transcription in vivo. Point mutations known to abrogate Mxr1p binding to AOX1 MXREs (Kranthi et al., 2009) abolish Mxrlp binding to $A L D-A$ MXREs as well. Analysis of GFP expression from $A L D-A$ promoters containing wild type or mutant MXREs indicate that atleast two MXREs are required for Mxrlp-mediated trans-activation of $A L D-A$ promoter in vivo. It is pertinent to note that the promoter of ACS1 encoding acetyl-CoA synthetase 1 consists of two MXREs, both of which are required for Mxrlp-mediated transactivation during acetate metabolism (37).

Of the four $A L D$ genes of $P$. pastoris, we have examined the function of $A L D-A$ and $A L D-D$ in this study. While deletion of ALD-A results in impaired growth in media containing ethanol and methanol, deletion of $A L D-D$ had no significant effect on the growth of cells metabolizing ethanol and methanol. Studies aimed at understanding the role of ALD-A during methanol metabolism indicate that ALD-A is essential for the utilization of methanol and it is required for the maintenance of normal levels of AOX protein in cells cultured in YNBM and YPM. ALD-A is neither a transcription factor nor it localizes to nucleus and therefore unlikely to regulate $A O X$ gene transcription. This is substantiated by the fact that only AOX protein but not mRNA levels are down regulated in $\Delta a l d-a$. Further, only AOX but not GFP expressed from $A O X 1$ promoter is down regulated in $\triangle a l d$ - $a$ indicating that $A O X 1$ promoter has no role in the ALD-A mediated down regulation of AOX. This is not a general property of all aldehyde dehydrogenases since AOX protein reduction is not seen in $\Delta a l d-d$. ALD-A and AOX localize to two different cellular compartments and therefore the down regulation of AOX protein is not due to direct protein-protein interactions. Formaldehyde generated from AOX-catalyzed reaction is converted to formate by formaldehyde dehydrogenase encoded by FLD1 and therefore a catalytic role for ALD-A in methanol metabolism is unlikely. Thus, the exact mechanism by which
AOX protein levels are depleted in $\Delta a l d-a$ needs further investigations.

This study has led to the identification of Mxr1p as a key regulator of $A L D-A$ which is essential for the metabolism of ethanol as well as methanol. Thus far, MXREs have been identified in genes involved in the metabolism of methanol, ethanol, glycerol, acetate and amino acids (28-34, $37,38,42,45$, this study) and Mxr1p has achieved a unique status as a global regulator of multiple metabolic pathways in K. phaffii (Fig. 6).

\section{EXPERIMENTAL PROCEDURES}

\section{Growth media and culture conditions}

Wild type (GS115, His-) strain of K. phaffii was a kind gift of James Cregg (28). K. phaffii KM71 strain (referred to as $\triangle A O X 1$ in this study) was purchased from Thermo Fisher Scientific. Cells were cultured in YP (1\% yeast extract, $2 \%$ peptone) medium consisting of $2 \%$ dextrose (YPD) or minimal media containing $0.17 \%$ yeast nitrogen base without amino acids and $0.5 \%$ ammonium sulfate (YNB) and $2 \%$ dextrose (YNBD), $1 \%$ methanol (YNBM) or $1 \%$ ethanol (YNBE). All components of cell culture media were purchased from Becton and Dickinson (BD) Biosciences. Yeast transformations were performed using Gene Pulser (Bio-Rad) as per manufacturer's instructions. For the isolation of recombinant plasmids, DH5 $\alpha$ strain of Escherichia coli was used. Expression of recombinant proteins was carried out using BL21(DE3) strain of E. coli. PEG method was used to prepare chemically component bacterial cells for transformation.

\section{Antibodies and other reagents}

Anti-AOX1 polyclonal antibodies and antiphosphoglycerate kinase (PGK) polyclonal antibodies have been described (43). Other antibodies used are: anti-MYC (Merck Millipore, OP-10), anti-GFP (Santa Cruz, SC-9996), Donkey, anti-mouse Alexa Flour 555 and 488 (A31570, A11001, Thermo Fisher). Western blotting was performed as described (43) and ImageJ software was applied to quantify all western blot data. Band intensity of the protein of interest was normalized to that of the loading control, PGK. Restriction enzymes, Taq DNA polymerase, and T4 DNA ligase were purchased from New England Biolabs (Frankfurt, Germany). Oligonucleotides were purchased from Sigma- 
Aldrich, India. Nucleotide sequence of primers used in $\mathrm{qPCR}$ and RT-PCR reactions will be provided on request.

\section{Subcellular localization studies}

Localization of AOX and ALD-A ${ }^{\text {Myc }}$ by immunofluorescence using anti-AOX and AntiMYC antibodies was carried out essentially as described (43). Fluorescent microscope (Leica DMLA) or confocal microscope Zeiss LSM 880 was used for visualization and capture of images.

\section{Quantitative real time PCR (qPCR)}

$K$. phaffii cells were cultured for 12-14 h and total RNA was isolated using RNA isolation kit (Cat. \# Z3100, Promega) as per manufacturer's instructions. cDNA was prepared and qPCR was carried out using iQ SYBR Green super mix and iQ5 multicolour real time PCR thermal cycler (Bio-Rad). The levels of mRNA expression relative to GS115 was normalized to $18 \mathrm{~S}$ rRNA. The comparative $\mathrm{Ct}$ method for relative quantification ( $\Delta \Delta \mathrm{Ct}$ method), which describes the change in expression of the target genes in a test sample relative to a calibrator sample, was used to analyze the data.

\section{Statistical analysis}

This was carried out essentially as described (43). Briefly, statistical tests such as student's t-test, one-way analysis of variance (ANOVA) followed by Tukey's multiple comparison were carried out using GraphPad Prism 5 software. Data are represented as mean \pm S.D. $P$ value summary is mentioned on the bar of each figure where * $P<0.05$; ** $P<0.005$; *** $P<0.0005$, ns, not significant.

\section{Mass spectrometry}

The protein bands of interest were excised precisely from SDS polyacrylamide gel and subjected to in-gel trypsin digestion using sequencing grade trypsin (Promega, USA). MALDI TOF was performed in HCT Ultra PTM Discovery System (ETD II-Bruker Daltonics) with 1100 series HPLC (Agilent). MASCOT protein mass fingerprint software with NCBI non-redundant database was used to identify target proteins.

\section{Electrophoretic mobility shift assay}

This was carried out with radiolabeled $A L D-A$ promoter regions and recombinant Mxrlp consisting of $\mathrm{N}$-terminal 150 amino acids $\left(\mathrm{Mxr1} \mathrm{p}^{\mathrm{N} 150}\right)$ as described (29).

\section{K. phaffii strains}

Amxr1: $\triangle m x r 1$ strains in which MXRl was disrupted by HIS4 or zeocin resistance cassette $\left(Z e o^{R}\right)$ have been described $(28,37)$.

$\Delta$ ald-a, $\Delta$ ald-d: A $0.911 \mathrm{~kb} A L D-A$ promoter region was amplified by PCR from $K$. phaffii genomic DNA using primer pair 5'GGACTGTTCAATTTGAAGTCGATGCTGAC G-3' and 5'-GCTATGGTGTGTGGGGGATCC GCACACGATCCCTTGGGAACTTGCGGTGG -3' (962 to 984 bp of GAPDH promoter in reverse complement, -89 to -114 bp of $A L D-A$ promoter in reverse complement). In another PCR, $1.2 \mathrm{~kb}$ of zeocin expression cassette was amplified by PCR from pGAPZA vector using the primer pair 5'CCACCGCAAGTTCCCAAGGGATCGTGTGC GGATCCCCCACACACCATAGC-3' (-89 to 114 bp of $A L D-A$ promoter , +962 to +984 bp of GAPDH promoter) and 5'GGAGTGTAAG CAATTCTGATAGCCTTGTGCCACATGTTG GTCTCCAGCTTG-3' (+1467 bp to +1493 bp in reverse complement of 3'-flanking region of $A L D$ $A,+2137$ to +2159 bp in reverse complement of $G A P D H$ promoter). In the third PCR, 872 bp of the 3 '-flanking region of $A L D$ - $A$ was amplified using the primer pair 5'-CAAGCTGGAGA CCAACATGTGAGCACAGGCTATCAGAATT GCTTACACTCC-3' (+2137 to +2159 bp of GAPDH promoter, +1467 to +1493 bp of region of $A L D-A$ gene) and 5'- GGAACTGGAG GCTTCCGCAGCAAACTCTC-3' (+2352 to +2381 bp in the reverse complement of 3'-flanking region of $A L D-A)$. All the three PCR products were purified and used as a template in the final PCR and amplified using a primer pair 5'GGACTGTTCAATTTGAAGTCGATGCTGAC G-3' and 5'-GGAACTGGAGGCTTCCGCAGC AAACTCTC-3' to obtain a $3.023 \mathrm{~kb}$ product consisting of zeocin expression cassette along with promoter and terminator of $A L D-A$. GS115 strain was transformed with $Z e o^{R}$ expression cassette and zeocin resistant colonies were selected. Deletion of $A L D-A$ was confirmed by PCR using genespecific primers. 
For the deletion of $A L D-D, 0.989 \mathrm{~kb}$ of promoter was amplified from $K$. phaffii genomic DNA by using primer set $1 \mathrm{~F}$ 5'CCAAAATGGTAACAACGTTCAAGTAAC-3' and 1R 5'-GCTATGGTGTGTGGGGGATC CGCACAAAGTGAAGGGGAAGATAAGAGC -3' (962 to 984 bp of GAPDH promoter in reverse complement, -9 to -32 bp of $A L D-D$ promoter in reverse complement). Zeo ${ }^{R}$ expression cassette of $1.2 \mathrm{~kb}$ was amplified from pGAPZA vector using the primer pair 2F 5'-GCTCTTATCTT CCCCTTCACTTTGTGCGGATCCCCCACAA CCATAGC-3' (9 to -32 bp of $A L D-D$ promoter and +962 to +984 bp of pGAPZA vector) and $2 \mathrm{R}$ 5'-GGCATATAGCAGGAGAGCTATTCCCTT TGCTCACATGTTGGTCTCCAGCTTG-3'

$(+1488$ bp to +1514 bp in reverse complement of 3 '-flanking region of $A L D-D,+2137$ to $+2159 \mathrm{bp}$ in reverse complement of pGAPZA vector). $1 \mathrm{~kb}$ terminator sequence or 3'-flanking region of $A L D$ $D$ promoter was amplified by using primer set $3 \mathrm{~F}$ 5'-CAAGCTGGAGACCAACATGTGAGCAAA GGGAATAGCTCTCCTGCTATATGCC-3'

$\left(+2137\right.$ to +2159 bp of $P_{G A P Z-A}$ vector, and $(+1488$ bp to $+1514 \mathrm{bp}$ of region of $A L D-D$ gene) and $3 \mathrm{R}$ 5'-TCAGAGACGATCTTCTCTTACGGG-3'

(reverse complement of +2465 to +2488 of $A L D$ $D$ terminator region). The final products of $A L D$ $D$ promoter, zeocin and $A L D-D$ terminator was purified and used as a template to make knockout construct of $A L D-D$. The final knockout construct was amplified by using primer set $1 \mathrm{~F}$ 5'CCAAAATGGTAACAACGTTCAAGTAAC-3' and 3R 5'- TCAGAGACGATCTTCTCTTA CGGG-3'. GS115 strain was transformed with the final PCR product and antibiotic resistant colonies were screened for zeocin resistance. Deletion of $A L D-D$ was confirmed by PCR by using genespecific primers.

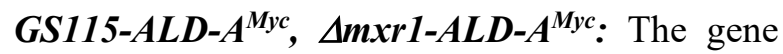
encoding ALD-A along with $1.0 \mathrm{~kb}$ of its promoter was cloned in- into pIB3 vector (\#25452, Addgene) and transformed into GS115 and $\Delta m x r 1$. The following primer pair was used: 5'TCCCCCCGGGATTGGAGAAGACAATGAAT CTG-3' and 5'-CCGCTCGAGCTACAGG TCTTCTTCAGAGTCAGTTTCTGTTCCTTAT GTCAGGAGTGTAAGC 3' (XmaI and XhoI sites in the primers are underlined). The reverse primer encoded the Myc tag. The PCR product was cloned into $p I B 3$ vector, the recombinant plasmid was linearized using SalI and transformed into GS115 and Amxrl. Recombinant clones were selected by plating on YNBD His plates and clones expressing Myc-tagged ALD-A (ALD$\mathrm{A}^{\mathrm{Myc}}$ ) were identified by western blotting using anti-Myc antibody.

GS115-P ALD-A $_{\text {GFP, }}$ Amxr1-P ALD-A $_{\text {GFP: The }}$ strains express GFP from $1.0 \mathrm{~kb}$ of $A L D-A$ promoter and were constructed as described below: Three different $\mathrm{PCR}$ reactions were carried out for the construction of $P_{A L D-A} G F P$ expression cassette. In the first PCR reaction, $1 \mathrm{~kb} A L D-A$ promoter was amplified from genomic DNA of GS115 strain of $K$. phaffii using a primer pair $1 \mathrm{~F}$ (5'CGGGATCCATTGGAGAAGACAATGAAT CTGAC-3', (BamHI site is underlined) and 1R (5'CTCCTTTACTAGTCAGATCTACCATGGA TAAAGGTAAGGGAAAAAAGCAAGTG-3') +1 to +25 bp of the gene encoding GFP and -1 to 28 bp of $\left.P_{A L D-A}\right)$. In the second PCR reaction, a 714 bp region of GFP gene was amplified from pREP41GFP vector using the primer pair 2F (5'CACTTGCTTTTTTCCCTTACCTTTATCCAT GGTAGATCTGACTAGTAAAGGAG-3', -1 to $28 \mathrm{bp}$ of $\left.P_{A L D-A}\right)$ and +1 to $+25 \mathrm{bp}$ of the gene encoding GFP) and $2 \mathrm{R}$ (5'CCGCTCGAGCTAGTGGTGGTGGCTAGCT

TTG-3') The XhoI site is underlined. In the third PCR reaction, the PCR products from the first two reactions were used as templates and amplified using the $1 \mathrm{~F}$ and $2 \mathrm{R}$ primers to get the $P_{A L D-A} G F P$ expression cassette, which was digested with BamHI and XhoI and cloned into pIB3 (\#25452, Addgene) to generate $p I B 3-P_{A L D-A} G F P$. The generated expression vectors were linearized with SalI and transformed into Komagataella phaffii GS115 and $\Delta m x r l$ strains and plated on YNBD$\mathrm{His}^{-}$agar plates, positive colonies were screened using anti-GFP antibody. GS115-ALD-A-M1 ${ }^{G F P,} \quad$ GS115-ALD-A-M2 ${ }^{G F P}$, GS115-ALD-A-M3 ${ }^{G F P}, \quad G S 115-A L D-A-M^{*} G F P$ and GS115-ALD-A-M2,3 ${ }^{\text {GFP }}$ : GS1 15 expressing GFP from $1.0 \mathrm{~kb} A L D-A$ promoter in which each of the three MXREs (M1, M2, M3), two MXREs and all the three MXRES $\left(\mathrm{M}^{*}\right)$ are mutated were 
generated as follows: $\quad P_{A L D-A} G F P$ plasmid with MXRE-M1 was generated as follows: $A L D-A$ promoter was PCR amplified using primer pair $1 \mathrm{~F}$ (5'-CGGGATCCATTGGAGAAGACAATGAA TCTGAC -3', (BamHI site is underlined) and 1R (5'-CCCTCTCCAAAAGAGGGACGATGTAG ATGAGAACCGTTGAGCGGAATCATGGGT G-3'). In another PCR, GFP was amplified using primer pair 2F (5'-CACCCATGATTC CGCTCAACGGTTCTCATCTACATCGTCCCT CTTTTGGAGAGGG-3' (mutation is underlined) and 2R (5'- CCGCTCGAGCTAGTGGTGGTG GCTAGCTTTG-3') (The XhoI site is underlined). In the third PCR reaction, the PCR products from the first two reactions were used as templates and amplified using the $1 \mathrm{~F}$ and $2 \mathrm{R}$ primers. Thereafter, the amplicon was digested with BamHI and XhoI and cloned into $p I B 3$ (Addgene, USA) to generate a plasmid carrying mutation in MXRE1 $\left(p P_{A L D-A} G F P-M 1\right)$. Similar strategy was used to generate the plasmids, $p P_{A L D-A} G F P-M 2 p P_{A L D-}$ ${ }_{A} G F P-M 3, p P_{A L D-A}-G F P-M 2,3$ and $p P_{A L D-A} G F P-$ $M^{*}$ carrying mutations in MXRE2 alone, MXRE3 alone or both MXRE 2 and 3 or all three MXREs respectively using appropriate $1 \mathrm{R}$ mutant reverse primers. Plasmids were linearized with SalI and transformed into GS115 to obtain the strains GS115- $A L D-A-M 1^{G F P}, \quad G S 115-A L D-A-M 2^{G F P}$, GS115-ALD-A-M3 ${ }^{G F P}, \quad G S 115-A L D-A-M 2,3^{G F P}$ and $G S 115-A L D-A-M^{*} G F P$ strains.

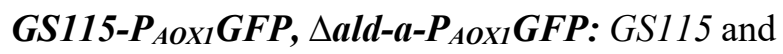
$\triangle$ ald- $a$ strains expressing GFP from $1.0 \mathrm{~kb}$ of $A O X 1$ promoter were generated as follows: $A O X 1$ promoter $(-1002 \mathrm{bp})$ was amplified by PCR using $K$. phaffii genomic DNA as a template and the primer pair 1F (5'CGGGGTACCTCATGTTGGTATTGTGAAAT AGACGCAGATC-3', -1002 to -971 bp of $\left.P_{A O X I}\right)$ and 1R (5'-CTCCTTTACTAGTCAGATCTA CCATCGTTTCGAATAATTAGTTGTTTTTTG ATC-3', +1 to +25 bp of the GFP ORF and -1 to 29 bp of $\left.P_{A O X I}\right)$. The $K p n I$ site is underlined. In the second PCR reaction, a 714 bp region of GFP gene was amplified from pREP41GFP vector using the primer pair 2F (5'- GATCAAAAAACAACTAA TTATTCGAAACGATGGTAGATCTGACTAG

TAAAGGAG-3', -1 to -29 bp of $P_{A O X I}$ and +1 to $+25 \mathrm{bp}$ of the GFP) and $2 \mathrm{R}$ (5'CCGCTCGAGCTAGTGGTGGTG GCTAGCT TTG-3') The XhoI site is underlined. In the third PCR reaction, the PCR products from the first two reactions were used as templates to amplify $P_{A O X I}$ GFP using $1 \mathrm{~F}$ and $2 \mathrm{R}$ primers. The final PCR product was digested with $K p n I$ and $X h o I$ and cloned into pIB3 vector (addgene, cat No. 25452) to yield $p I B 3-P_{A O X I} G F P$. The recombinant plasmid was linearized with SalI and transformed into GS115 and $\Delta$ ald-a strains. GFP expressing clones were identified by western blotting using anti-GFP antibodies.

\section{Conflict of interest}

The authors declare that they have no conflicts of interest with the contents of this article.

Data availability statement: All data presented and discussed are contained within the manuscript.

\section{Footnotes / Funding}

This work was supported by the J. C. Bose Fellowship grant SB/S2/JCB-025/2015 awarded by the Science and Engineering Research Board, New Delhi, India and the research grant BT/PR30986/BRB/10/1751/2018 awarded by the Department of Biotechnology, New Delhi, India (to P.N.R). Funding from the Department of Science and Technology Fund for Improvement of S\&T Infrastructure in Higher Educational Institutions (DST-FIST), the University Grants Commission and the Department of Biotechnology (DBT)-Indian Institute of Science partnership program is acknowledged. K.K.R is a recipient of junior and senior research fellowship of Indian Council Medical Research, New Delhi, India. 


\section{REFERENCES}

1. Lutstorf, U., and Megnet, R. (1968) Multiple forms of alcohol dehydrogenase in Saccharomyces cerevisiae. I. Physiological control of ADH-2 and properties of ADH-2 and ADH-4. Arch Biochem Biophys. 126, 933-944

2. Bennetzen, J.L., and Hall, B.D. (1982) The primary structure of the Saccharomyces cerevisiae gene for alcohol dehydrogenase. J. Biol. Chem. 257, 3018-25

3. Russell, D.W., Smith, M., Williamson, V.M., and Young, E.T. (1983) Nucleotide sequence of the yeast alcohol dehydrogenase II gene. J. Biol. Chem. 258, 2674-82

4. Denis, C.L., Ciriacy, M., and Young, E.T. (1981) A positive regulatory gene is required for accumulation of the functional messenger RNA for the glucose-repressible alcohol dehydrogenase from Saccharomyces cerevisiae. J. Mol. Biol. 148, 355-68

5. Ciriacy, M. (1975) Genetics of alcohol dehydrogenase in Saccharomyces cerevisiae. II. Two loci controlling synthesis of the glucose-repressible ADH II. Mol. Gen. Genet. 138, 157-64

6. Ciriacy, M. (1979) Isolation and characterization of further Cis- and Trans-acting regulatory elements involved in the synthesis of glucose-repressible alcohol dehydrogenase (ADHII) in Saccharomyces cerevisiae. MGG Mol. Gen. Genet. 176, 427-431

7. Young, E.T., and Pilgrim, D. (1985) Isolation and DNA sequence of ADH3, a nuclear gene encoding the mitochondrial isozyme of alcohol dehydrogenase in Saccharomyces cerevisiae. Mol. Cell. Biol. 5, 3024-3034

8. Drewke, C., and Ciriacy, M. (1988) Overexpression, purification and properties of alcohol dehydrogenase IV from Saccharomyces cerevisiae. Biochim. Biophys. Acta. 950, 54-60

9. Leskovac, V., Trivic, S., and Pericin, D. (2002) The three zinc-containing alcohol dehydrogenases from baker's yeast, Saccharomyces cerevisiae. FEMS Yeast Res. 2, 481-494

10. Smith, M.G., Des Etages, S.G., and Snyder, M. (2004) Microbial synergy via an ethanol-triggered pathway. Mol. Cell. Biol. 24, 3874-84

11. Dickinson, J.R., Salgado, L.E.J., and Hewlins, M.J.E. (2003) The catabolism of amino acids to long chain and complex alcohols in Saccharomyces cerevisiae. J. Biol. Chem. 278, 8028-8034

12. Wang, X., Mann, C.J., Bai, Y., Ni, L., and Weiner, H. (1998) Molecular cloning, characterization, and potential roles of cytosolic and mitochondrial aldehyde dehydrogenases in ethanol metabolism in Saccharomyces cerevisiae. J. Bacteriol. 180, 822-30

13. Navarro-Aviño, J.P., Prasad, R., Miralles, V.J., Benito, R.M., and Serrano, R. (1999) A proposal for nomenclature of aldehyde dehydrogenases in Saccharomyces cerevisiae and characterization of the stress-inducible ALD2 andALD3 genes. Yeast. 15, 829-842

14. Aranda, A., and del Olmo, M. 1. (2003) Response to acetaldehyde stress in the yeast Saccharomyces cerevisiae involves a strain-dependent regulation of several ALD genes and is mediated by the general stress response pathway. Yeast. 20,747-759

15. Denis, C.L., and Young, E.T. (1983) Isolation and characterization of the positive regulatory gene ADR1 from Saccharomyces cerevisiae. Mol. Cell. Biol. 3, 360-370

16. Walther, K., and Schuller, H.-J. (2001) Adr1 and Cat8 synergistically activate the glucoseregulated alcohol dehydrogenase gene $\mathrm{ADH} 2$ of the yeast Saccharomyces cerevisiae. Microbiology. 147, 2037-2044

17. Simon, M., Adam, G., Rapatz, W., Spevak, W., and Ruis, H. (1991) The Saccharomyces cerevisiae ADR1 gene is a positive regulator of transcription of genes encoding peroxisomal proteins. Mol. Cell. Biol. 11, 699-704

18. Tachibana, C., Yoo, J.Y., Tagne, J.B., Kacherovsky, N., Lee, T.I., and Young, E.T. (2005) Combined Global Localization Analysis and Transcriptome Data Identify Genes That Are Directly Coregulated by Adr1 and Cat8. Mol. Cell. Biol. 25, 2138-2146

19. Cregg, J.M., Cereghino, J.L., Shi, J., and Higgins, D.R. (2000) Recombinant protein expression in Komagataella phaffii. Appl. Biochem. Biotechnol. - Part B Mol. Biotechnol. 16, 23-52

20. Vanz, A.L., Lünsdorf, H., Adnan, A., Nimtz, M., Gurramkonda, C., Khanna, N., and Rinas, U. (2012) Physiological response of Komagataella phaffii GS115 to methanol-induced high level 
production of the Hepatitis B surface antigen: catabolic adaptation, stress responses, and autophagic processes. Microb. Cell Fact. 11, 103

21. Nocon, J., Steiger, M.G., Pfeffer, M., Sohn, S.B., Kim, T.Y., Maurer, M., Rubmayer, H., Pflugl, S., Ask, M., Haberhauer-Troyer, C., Ortmayr, K., Hann, S., Koellensperger, G., Gasser, B., Lee, S.Y., and Mattanovich, D. (2014) Model based engineering of Komagataella phaffii central metabolism enhances recombinant protein production. Metab. Eng. 24, 129-138

22. De Schutter, K., Lin, Y.C., Tiels, P., Van Hecke, A., Glinka, S., Weber-Lehmann, J., Rouze, P., Van de Peer,Y., and Callewaert, N. (2009) Genome sequence of the recombinant protein production host Komagataella phaffii. Nat. Biotechnol. 27, 561-566

23. Mattanovich, D., Graf, A., Stadlmann, J., Dragosits, M., Redl, A., Maurer, M., Kleinheinz, M., Sauer, M., Altmann, F., and Gasser, B. (2009) Genome, secretome and glucose transport highlight unique features of the protein production host Komagataella phaffii. Microb. Cell Fact. 8, 29

24. Küberl, A., Schneider, J., Thallinger, G.G., Anderl, I., Wibberg, D., Hajek, T., Jaenicke, S., Brinkrolf, K., Goesmann, A., Szczepanowski, R., Puhler, A., Schwab, H., Glieder, A., and Pichler, H. (2011) High-quality genome sequence of Komagataella phaffii CBS7435. J. Biotechnol. 154, 312-320

25. Karaoglan, M., Karaoglan, F.E., and Inan, M. (2016) Functional analysis of alcohol dehydrogenase (ADH) genes in Komagataella phaffii. Biotechnol. Lett. 38, 463-469

26. Karaoglan, M., Karaoglan, F.E., and Inan, M. (2016) Comparison of ADH3 promoter with commonly used promoters for recombinant protein production in Komagataella phaffii. Protein Expr. Purif. 121, 112-117

27. Ohsawa, S., Nishida, S., Oku, M., Sakai, Y., and Yurimoto, H. (2018) Ethanol represses the expression of methanol-inducible genes via acetyl-CoA synthesis in the yeast Komagataella phaffii. Sci. Rep. 8, 18051

28. Lin-Cereghino, G.P., Godfrey, L., de la Cruz, B.J., Johnson, S., Khuongsathiene, S., Tolstorukov, I., Yan, M., Lin-Cereghino, J., Veenhuis, M., Subramani, S., and Cregg, J.M. (2006) Mxr1p, a Key Regulator of the Methanol Utilization Pathway and Peroxisomal Genes in Komagataella phaffii. Mol. Cell. Biol. 26, 883-897

29. Kranthi, B.V., Kumar, R., Kumar, N.V., Rao, D.N., and Rangarajan, P.N. (2009) Identification of key DNA elements involved in promoter recognition by Mxr1p, a master regulator of methanol utilization pathway in Komagataella phaffii. Biochim. Biophys. Acta-Gene Regul. Mech. 1789, 460-468

30. Kranthi, B.V., Vinod Kumar, H.R., and Rangarajan, P.N. (2010) Identification of Mxr1p-binding sites in the promoters of genes encoding dihydroxyacetone synthase and peroxin 8 of the methylotrophic yeast Komagataella phaffii. Yeast. 27, 705-711

31. Kumar, N.V., and Rangarajan, P.N. (2012) The Zinc Finger Proteins Mxr1p and Repressor of Phosphoenolpyruvate Carboxykinase (ROP) Have the Same DNA Binding Specificity but Regulate Methanol Metabolism Antagonistically in Komagataella phaffii. J. Biol. Chem. 287, 34465-34473.

32. Sahu, U., Krishna Rao, K., and Rangarajan, P.N. (2014) Trm1p, a Zn(II)2Cys6-type transcription factor, is essential for the transcriptional activation of genes of methanol utilization pathway, in Komagataella phaffii. Biochem. Biophys. Res. Commun. 451, 158-164

33. Wang, X., Cai, M., Shi, L., Wang, Q., Zhu, J., Wang, J., Zhou, M., Zhou, X., and Zhang, Y. (2016) $\mathrm{PpNrg} 1$ is a transcriptional repressor for glucose and glycerol repression of AOX1 promoter in methylotrophic yeast Komagataella phaffii. Biotechnol. Lett. 38, 291-8

34. Shi, L., Wang, X., Wang, J., Zhang, P., Qi, F., Cai, M., Zhang, Y., and Zhou, X. (2018) Transcriptome analysis of $\Delta$ mig $1 \Delta$ mig2 mutant reveals their roles in methanol catabolism, peroxisome biogenesis and autophagy in methylotrophic yeast Komagataella phaffii. Genes Genomics, 40, 399-412

35. Vogl, T., Sturmberger, L., Fauland, P.C., Hyden, P., Fischer, J.E., Schmid, C., Thallinger, G.G., Geier, M., and Glieder, A. (2018) Methanol independent induction in Komagataella phaffii by 
simple derepressed overexpression of single transcription factors. Biotechnol. Bioeng. 115, 10371050

36. Wang, J., Wang, X., Shi, L., Qi, F., Zhang, P., Zhang, Y., Zhou, X., Song, Z., and Cai, M. (2017) Methanol-Independent Protein Expression by AOX1 Promoter with trans-Acting Elements engineering and Glucose-Glycerol-Shift Induction in Komagataella phaffii. Sci. Rep. 7, 41850

37. Sahu, U., and Rangarajan, P.N. (2016) Regulation of Acetate Metabolism and Acetyl Co-a Synthetase 1 (ACS1) Expression by Methanol Expression Regulator 1 (Mxr1p) in the Methylotrophic Yeast Komagataella phaffii. J. Biol. Chem. 291, 3648-3657

38. Sahu, U., and Rangarajan, P.N. (2016) Methanol Expression Regulator 1 (Mxr1p) Is Essential for the Utilization of Amino Acids as the Sole Source of Carbon by the Methylotrophic Yeast, Komagataella phaffii. J. Biol. Chem. 291, 20588-20601

39. Cardarelli, S., D’Amici, S., Tassone, P., Tramonti, A., Uccelletti, D., Mancini, P., and Saliola, M. (2016) Characterization of the transcription factor encoding gene, K1ADR1: Metabolic role in Kluyveromyces lactis and expression in Saccharomyces cerevisiae. Microbiol. (United Kingdom). 162, 1933-1944

40. Ramírez, M.A., and Lorenz, M.C. (2009) The transcription factor homolog CTF1 regulates $\beta$ oxidation in Candida albicans. Eukaryot. Cell. 8, 1604-1614

41. van der Klei IJ., Harder, W., and Veenhuis M. (1991) Biosynthesis and assembly of alcohol oxidase, a peroxisomal matrix protein in methylotrophic yeasts: a review. Yeast. 7, 195-209.

42. Parua, P.K., Ryan, P.M., Trang, K., and Young,E.T. (2012) Komagataella phaffii 14-3-3 regulates transcriptional activity of the methanol inducible transcription factor Mxr1 by direct interaction. Mol. Microbiol. 85, 282-98

43. Dey, T., Krishna Rao, K., Khatun, J., and Rangarajan, P.N. (2018) The nuclear transcription factor Rtg1p functions as a cytosolic, post-transcriptional regulator in the methylotrophic yeast Komagataella phaffii. J. Biol. Chem. 293, 16647-16660

44. Uppada, V., and Noronha, SB. (2016) Functional characterization of alcohol dehydrogenase from Crabtree-negative yeast Komagataella (Pichia) pastoris. Curr. Sci. 111, 1596-1598

45. Li, X., Yang, Y., Zhan, C., Zhang, Z., Liu, X., Liu, H., and Bai, Z. (2018) Transcriptional analysis of impacts of glycerol transporter 1 on methanol and glycerol metabolism in Komagataella phaffii. FEMS Yeast Res. 18, doi.org/10.1093/femsyr/fox081

46. Ergün, B.G., Gasser, B., Mattanovich, D., and Çalık, P. (2019) Engineering of alcohol dehydrogenase 2 hybrid-promoter architectures in Komagataella phaffii to enhance recombinant protein expression on ethanol. Biotechnol. Bioeng. 116, 2674-2686

47. Saint-Prix, F., Bönquist, L., and Dequin, S (2004) Functional analysis of the ALD gene family of Saccharomyces cerevisiae during anaerobic growth on glucose: the $\mathrm{NADP}+$-dependent Ald6p and Ald5p isoforms play a major role in acetate formation. Microbiology. 150, 2209-2220. 
Table.1. K. phaffii alcohol dehydrogenase (ADHs) and aldehyde dehydrogenases (ALDs).

\section{K. phaffii gene Designation in this study References}

\begin{tabular}{|c|c|c|}
\hline \multicolumn{3}{|l|}{$\overline{A D H:}$} \\
\hline PAS_chr3_0006 & ADH-A (XP_002492217.1; XM_002492172.1) & 44 \\
\hline PAS_chr1-1_0357 & ADH-B (XP_002490014.1; XM_002489969.1) & 44 \\
\hline PAS_chr2-1_0472 & ADH-C ${ }^{*}$ (XP_002491382.1; XM_002491337.1) & $26,27,45,46$ \\
\hline PAS_chr4_ $0 \overline{5} 76$ & ADH-D ${ }^{@}\left(X \bar{P} \_002494014.1 ; X \bar{M} \_002493969.1\right)$ & 45 \\
\hline PAS-chr3-0349 & ADH-E (XP_002492569.1; XM_002492524) & 44 \\
\hline PAS-chr2-1_0313 & ADH-F (XP_002491208.1; XM_002491163.1) & 44 \\
\hline \multicolumn{3}{|c|}{ (6) } \\
\hline PAS_chr4_0043 & ALD-A (XP_002493450.1) (XM_002493405.1) & This study \\
\hline PAS_chr3_0987 & ALD-B $^{\#}\left(\mathrm{XP}_{-}^{-}\right.$002493229.1) (XM_002493184.1) & 45 \\
\hline PAS_chr2-1_0853 & $\mathrm{ALD}^{+} \mathrm{C}^{+}(\mathrm{XP}-002491418.1)\left(\mathrm{XM}{ }^{-} 002491373.1\right)$ & 27,45 \\
\hline PAS_chr2-1_0453 & ALD-D $^{\$}\left(X^{-} \_002491360.1\right)\left(X M^{-}\right.$002491315.1) & 45 \\
\hline \multicolumn{3}{|c|}{ 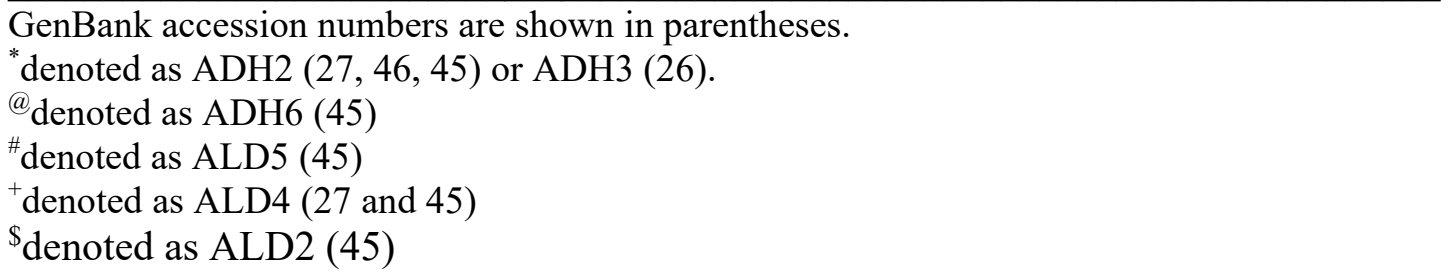 } \\
\hline
\end{tabular}


Table 2. K. phaffii strains used in this study

\begin{tabular}{|c|c|c|}
\hline Strain & Genotype & References \\
\hline GS115 & his4 & 28 \\
\hline KM71 ( $\Delta$ aox 1$)$ & Aaoxl:ARG4 his4 arg4 & ThermoFisher \\
\hline$\Delta m x r 1$ & GS115, Ppmxr1D::His4 & 28 \\
\hline$\Delta m x r 1$ & GS115, Ppmxr1D::Zeocin ${ }^{r}$ & 37 \\
\hline$\Delta$ ald-a & GS115, Ppald-a $\Delta::$ Zeocin $^{r}$ & This study \\
\hline$\Delta$ ald-d & GS115, Ppald-d $\Delta::$ Zeocin $^{r}$ & This study \\
\hline$G S 115-A L D-A^{M y c}$ & $G S 115, H_{i s} 4^{+}:\left(P_{A L D-A} P p A L D-A^{M y c}\right)$ & This study \\
\hline$\Delta m x r 1-A L D-A^{M y c}$ & $\Delta m x r 1, H i s 4^{+} \because\left(P_{A L D-A} P p A L D-A^{M y c}\right)$ & This study \\
\hline$G S 115-P_{A L D-A^{-}} G F P$ & $G S 115, H_{i S} 4^{+}::\left(P_{A L D-A} G F P\right)$ & This study \\
\hline$\Delta m x r 1-P_{A L D-A^{-}} G F P$ & $\Delta m x r 1, H_{i s} 4^{+}::\left(P_{A L D-A} G F P\right)$ & This study \\
\hline$G S 115-A L D-A-M 1^{G F P}$ & $G S 115, H i s 4^{+}: \because\left(P_{A L D-A-M I} G F P\right)$ & This study \\
\hline$G S 115-A L D-A-M 2^{G F P}$ & $G S 115, \mathrm{HiS}^{+}: \because\left(P_{A L D-A-M 2} G F P\right)$ & This study \\
\hline$G S 115-A L D-A-M 2,3^{G F P}$ & GS115, His $4^{+} \because:\left(P_{A L D-A-M 2} G F P\right)$ & This study \\
\hline$G S 115-A L D-A-M 3^{G F P}$ & $G S 115, \mathrm{HiS}^{+}: \because\left(P_{A L D-A-M 3} G F P\right)$ & This study \\
\hline$G S 115-A L D-A-M^{*} G F P$ & $G S 115, H_{i S} 4^{+}:\left(P_{A L D-A-M^{*}} G F P\right)$ & This study \\
\hline$G S 115-P_{A O X 1}-G F P$ & $G S 115, H_{i S} 4^{+}:\left(P_{A O X I} G F P\right)$ & This study \\
\hline$\Delta$ ald $-a-P_{A O X I}-G F P$ & $\Delta$ ald-a, His $4^{+}::\left(P_{A O X I} G F P\right)$ & This study \\
\hline
\end{tabular}




\section{Figures and Legends}

Fig.1. Identification of $A L D-A$ as the target of Mxr1p and analysis of its function during ethanol and methanol metabolism. A. Analysis of growth of GS115 and $\triangle m x r 1$ cultured in YNBE and YPE. B. Schematic representation of ethanol utilization pathway of $S$. cerevisiae. $S$. cerevisiae genome encodes $\mathrm{NAD}^{+}$as well as NADP ${ }^{+}$ALDs (47). C. Quantitation of mRNAs encoding ADHs and ALDs of $K$. phaffii by qPCR in GS115 cultured in YNBD and YNBE. D. Quantitation of mRNAs encoding ADHs and ALDs of $K$. phaffii by qPCR in GS115 and $\triangle m x r l$ cultured in YNBE. Error bars in each figure indicate S.D. Data is from three biological replicates $(n=3)$. In the graphs, $P$ value summary is mentioned on the bar of each figure. ${ }^{*} \mathrm{p}<0.05, * * \mathrm{p}<0.005, * * * \mathrm{p}<0.0005, \mathrm{~ns}$, not significant. Student's paired or unpaired t-test was done. E. Quantitation of $A L D-A$ mRNA levels by qPCR in GS115 and $\triangle m x r l$ cultured in YNBM and YNBD. Error bars in each figure indicate S.D. Data is from three biological replicates $(n=3)$. In the graphs, $P$ value summary is mentioned on the bar of each figure. ${ }^{*} p<0.05, * * p<0.005, * * * p<0.0005$, ns, not significant. Student's paired or unpaired t-test was done. F. Strategy for the generation of $\Delta a l d-a$. G. Confirmation of deletion of $A L D-A$ by PCR. $A L D-A$ was amplified from genomic DNA by PCR using gene-specific primers. H. Analysis of growth of GS115 and $\triangle m x r l$ cultured in YNBE and YNBM.

Fig. 2. Confirmation of Mxrlp as a regulator of ALD-A expression. A. Analysis of ALD-A ${ }^{\mathrm{Myc}}$ levels in GS115 and $\triangle m x r 1$ in cells cultured in YNBE, YNBM and YNBD by western blotting using anti-Myc antibodies. M, protein molecular weight markers $(\mathrm{kDa})$. Quantitation of the data is presented. The intensity of individual bands was quantified and expressed as arbitrary units \pm S.D. relative to controls. Data is from three biological replicates $(\mathrm{n}=3)$. B. Schematic representation of $P_{A L D-A} G F P$ and analysis of GFP levels in GS115 and $\triangle m x r l$ cultured in YNBE and YNBM by western blotting using anti-GFP antibodies. PGK was used as loading control. M, protein molecular weight markers $(\mathrm{kDa})$. Quantitation of the data presented in $\mathrm{B}$ is also shown. The intensity of individual bands was quantified and expressed as arbitrary units \pm S.D. relative to controls. Data is from three biological replicates $(n=3)$. C. Subcellular localization of ALD$\mathrm{A}^{\mathrm{Myc}}$ as analyzed by immunofluorescence using confocal microscopy. Mouse anti-Myc antibodies and Alexa Flour 488-conjugated, donkey anti-mouse antibodies were used. DAPI was used to stain the nucleus.

Fig.3. Identification of MXREs in $A L D-A$ promoter. A. Schematic representation of position of putative MXREs in $A L D-A$ promoter. B. Nucleotide sequence of oligonucleotides used in EMSA. MXREs are boxed and point mutations within MXREs are underlined. C. Analysis of recombinant Mxrlp ${ }^{\mathrm{N} 150}$ binding to radiolabeled $A L D-A$ promoter regions by EMSA. Point mutations within MXREs abrogate Mxrlp binding.

Fig.4. Analysis of function of MXREs of ALD-A promoter in vivo. A. Schematic representation of different $P_{A L D-A} G F P$ constructs. B. Analysis of GFP expression from $A L D-A$ promoter containing wild type or mutant MXREs by western blotting using anti-GFP antibodies in cells cultured in YNBE and YNBM. PGK was used as loading control. M, protein molecular weight markers $(\mathrm{kDa})$. Quantitation of the western blot data is also shown. The intensity of individual bands was quantified and expressed as arbitrary units \pm S.D. relative to controls Data is from three biological replicates $(\mathrm{n}=3)$. C. Schematic representation of $P_{A L D-A} G F P$ construct carrying two mutant MXREs. D. Analysis of GFP expression from $A L D-A$ promoter containing wild type or mutant MXREs by western blotting using anti-GFP antibodies in cells cultured in YNBE and YNBM. PGK was used as loading control. M, protein molecular weight markers (kDa). Quantitation of the western blot data is also shown. The intensity of individual bands was quantified and expressed as arbitrary units \pm S.D. relative to controls. Data is from three biological replicates $(n=3)$. E. Schematic representation of regulation of $A L D-A$ by Mxr1p. during ethanol and methanol metabolism.

Fig. 5. Regulation of AOX protein levels by ALD-A in cells cultured in YNBM. A. Analysis of AOX protein levels in GS115 and $\Delta a l d-a$ cultured in YNBM by SDS-PAGE. Gels were stained with Coomassie 
Brilliant blue R. Protein molecular weight markers $(\mathrm{kDa})$ are indicated $(\mathrm{M})$. B. Analysis of AOX protein levels in GS115 and $\Delta a l d-a$ cultured in YNBM by western blotting. Anti-AOX antibodies were used. M, protein molecular weight markers. Quantification of intensity of bands is shown. Data is from three biological replicates $(\mathrm{n}=3)$. C. Quantification of $A O X 1 \mathrm{mRNA}$ levels by qPCR. Error bars indicate S.D. $\mathrm{n}=3$. ns, not significant. D. Quantification of AOX and GFP expressed from $A O X I$ promoter by western blotting using anti-AOX and anti-GFP antibodies respectively. PGK was used as loading control. Lysates were prepared from GS115P $A O X^{-} G F P$ strain in which AOX protein is synthesized from the $A O X 1$ and $A O X 2$ loci while GFP is synthesized from $1.0 \mathrm{~kb} A O X$ promoter of the $P_{A O X I}-G F P$ expression cassette integrated at His4 locus. E. Subcellular localization of ALD-A ${ }^{\mathrm{Myc}}$ and AOX as analyzed by immunofluorescence by confocal microscopy using rabbit anti-Myc antibodies and mouse anti-AOX antibodies respectively. DAPI was used to stain the nucleus. F. Analysis of AOX protein levels in GS115 and Aald-d cultured in cells cultured in YNBM and YPM by SDS-PAGE. Gels were stained with Coomassie Brilliant blue R. M, protein molecular weight markers (kDa). G. Analysis of growth of GS115 and Aald-d cultured in YNBE and YNBM. H. Schematic representation of regulation of AOX protein levels by ALD-A during methanol metabolism. I. Schematic representation of regulation of AOX expression during methanol metabolism. Mxrlp regulates $A O X 1$ and $A L D-A$ expression at the transcriptional level and ALD-A in turn regulates AOX protein levels at the post-transcriptional level.

Fig. 6. Schematic representation depicting Mxr1p as a global regulator of multiple metabolic pathways of $K$. phaffii. Target genes of Mxrlp containing MXREs in their promoters are shown (28-34, 37, 38, 42, 45, this study). 
A

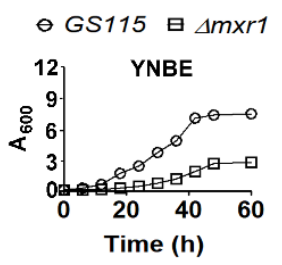

B

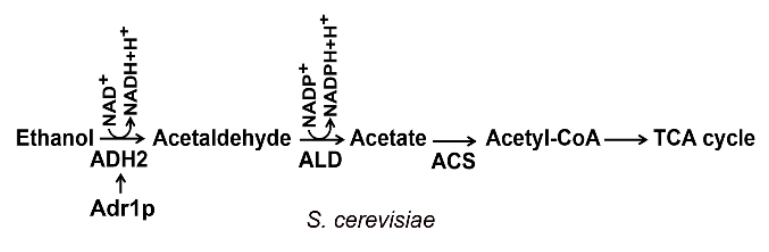

C
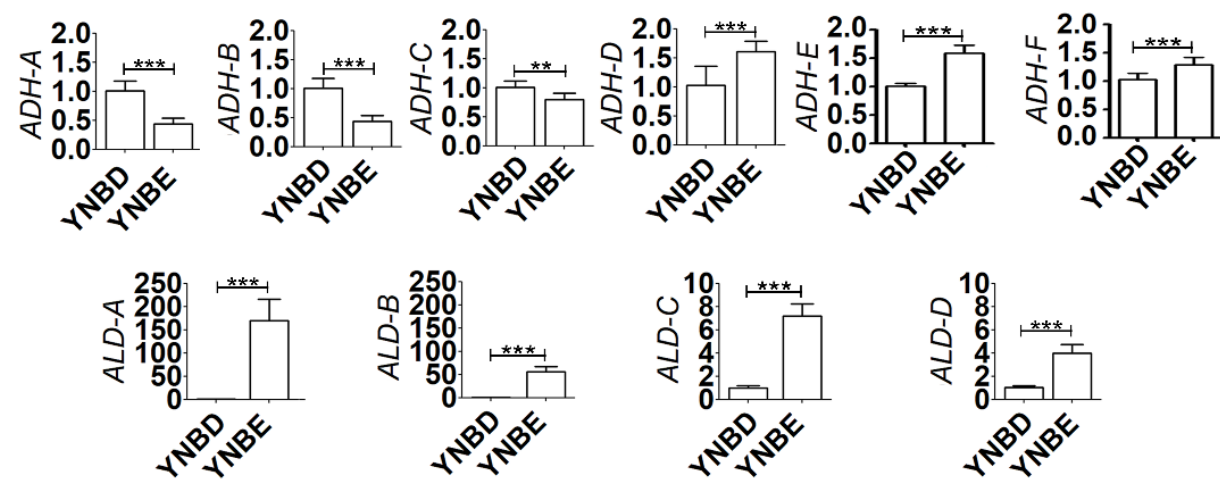

D

\section{口GS115 口 $\Delta m \times r 1$}

YNBE

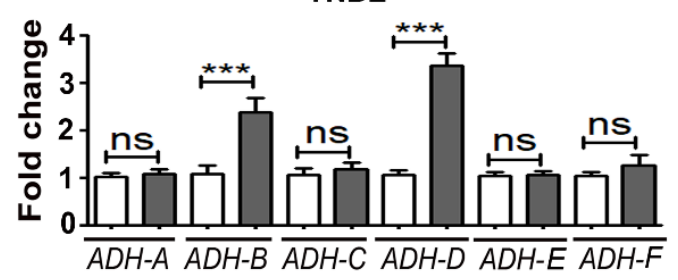

口GS115 口Amxr1

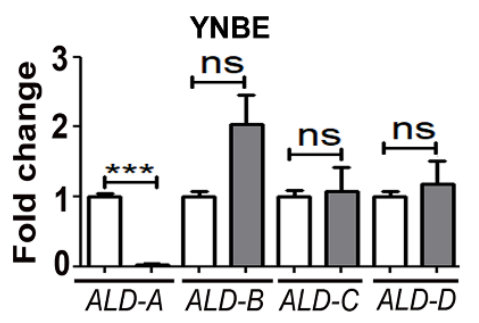

E

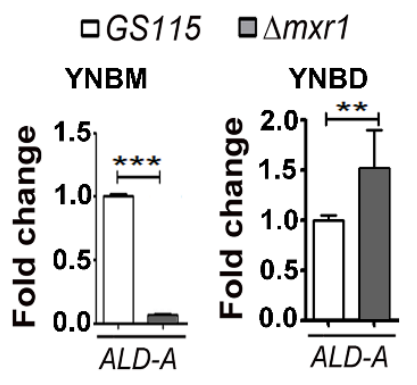

F

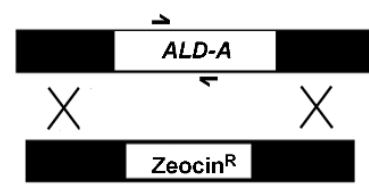

G

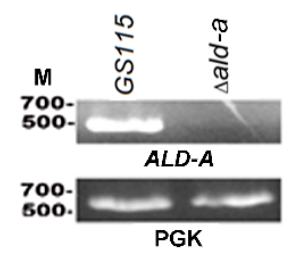

Fig. 1
$\mathrm{H}$

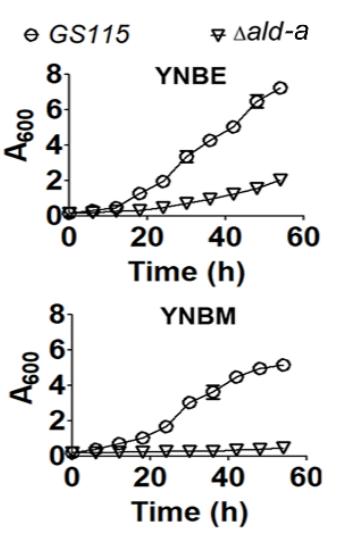


A

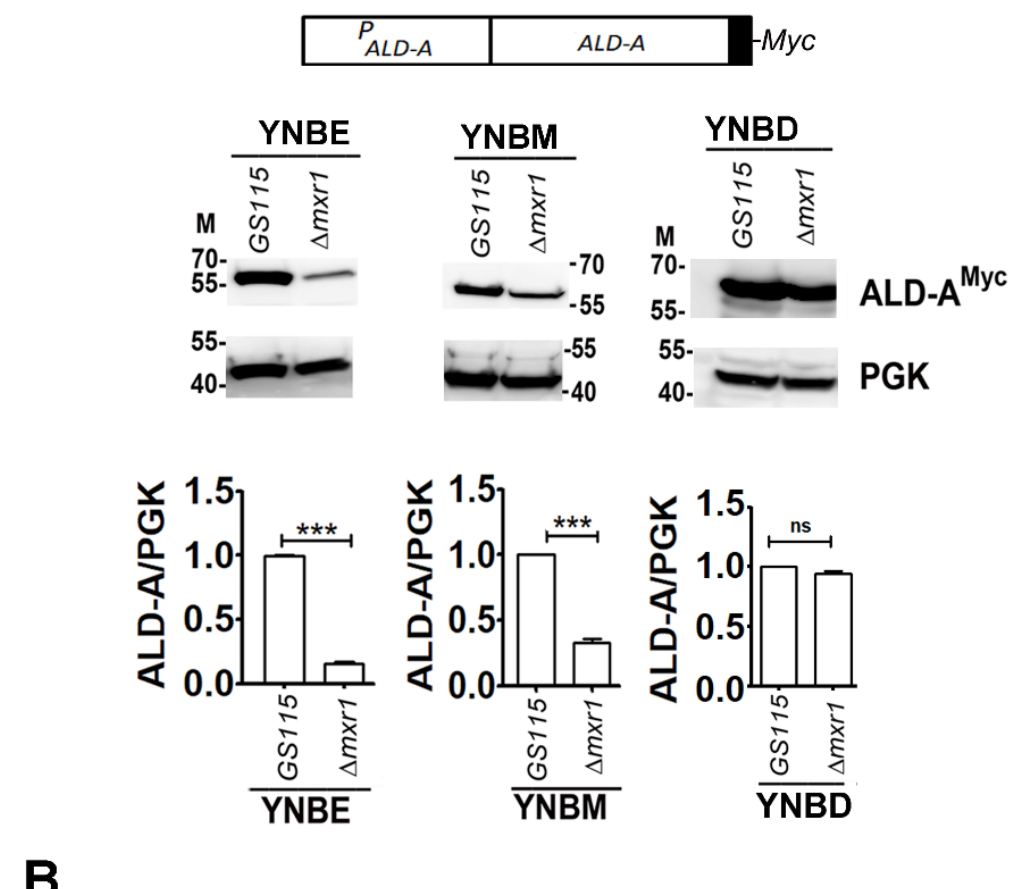

B
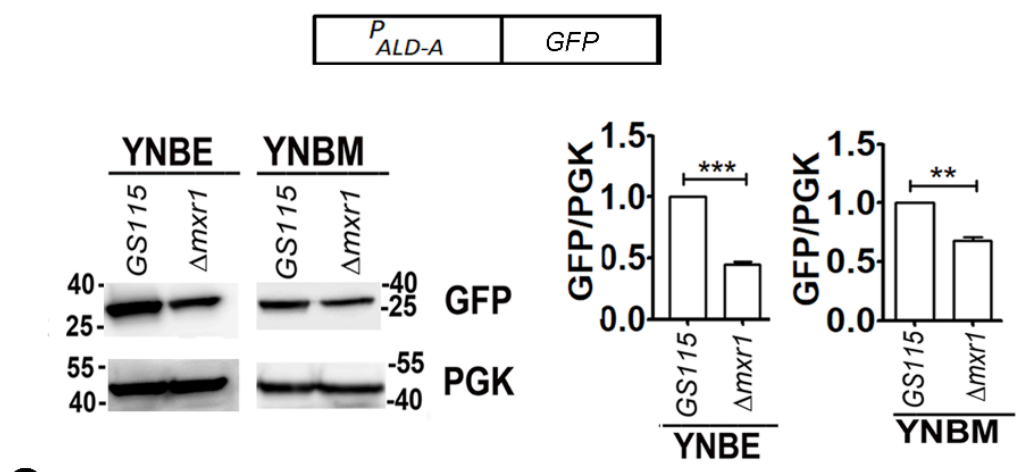

C

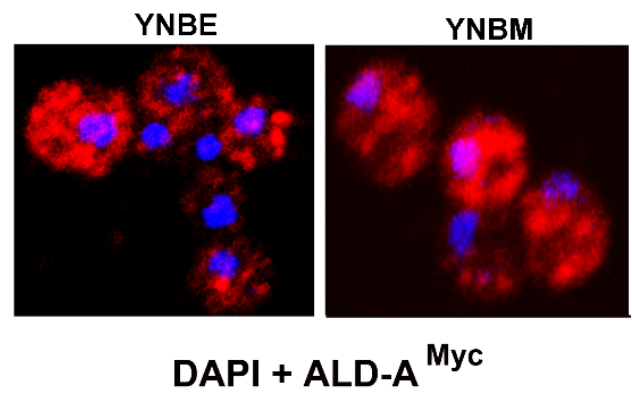

Fig. 2 
A

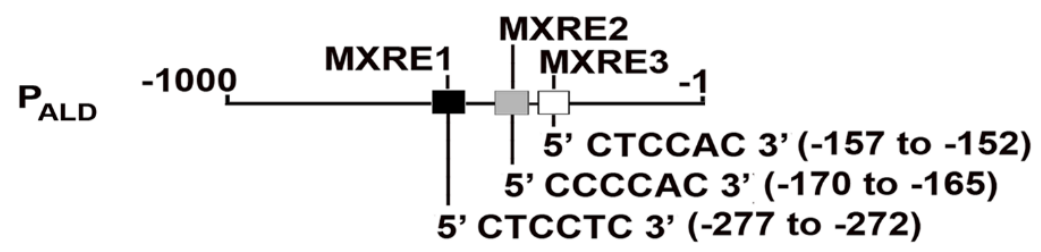

B

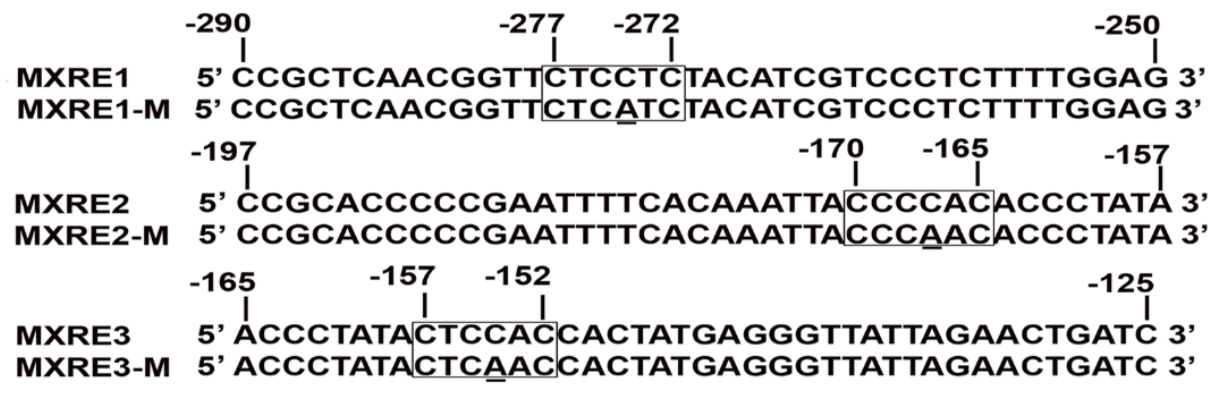

C

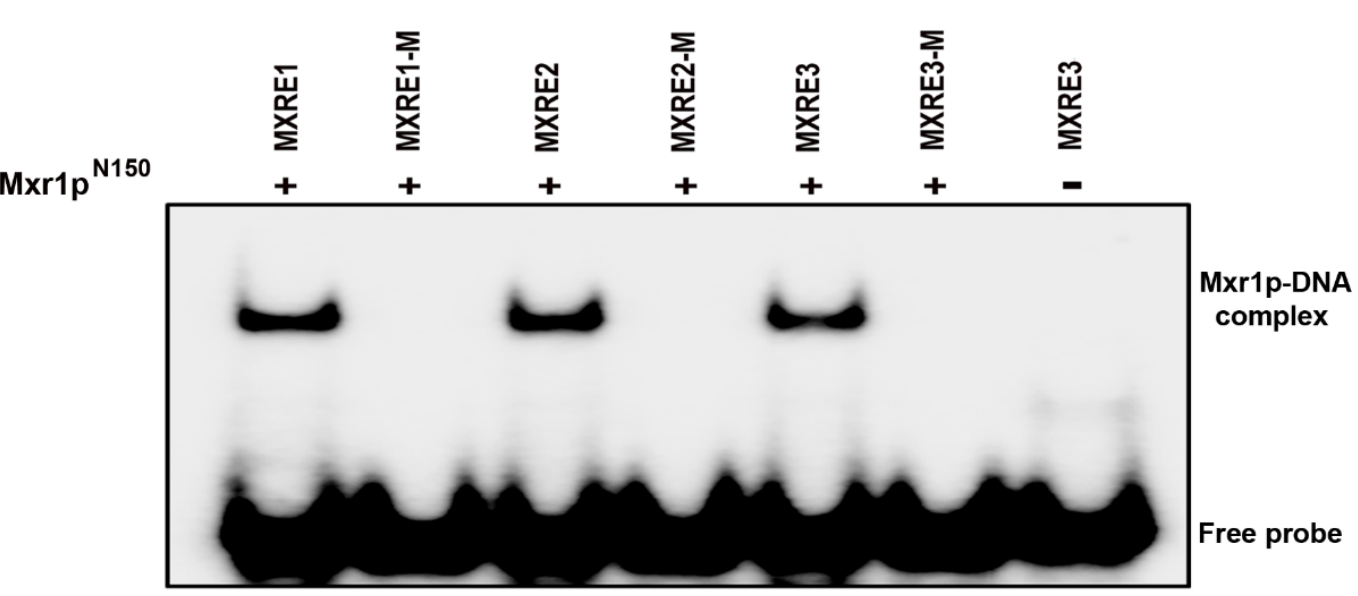

Fig. 3 
A

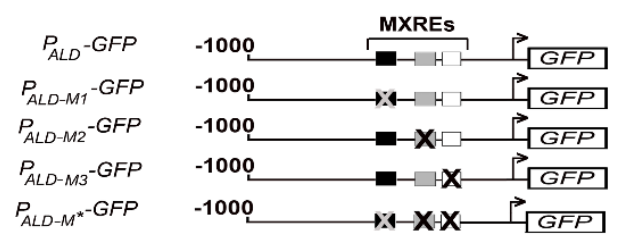

B
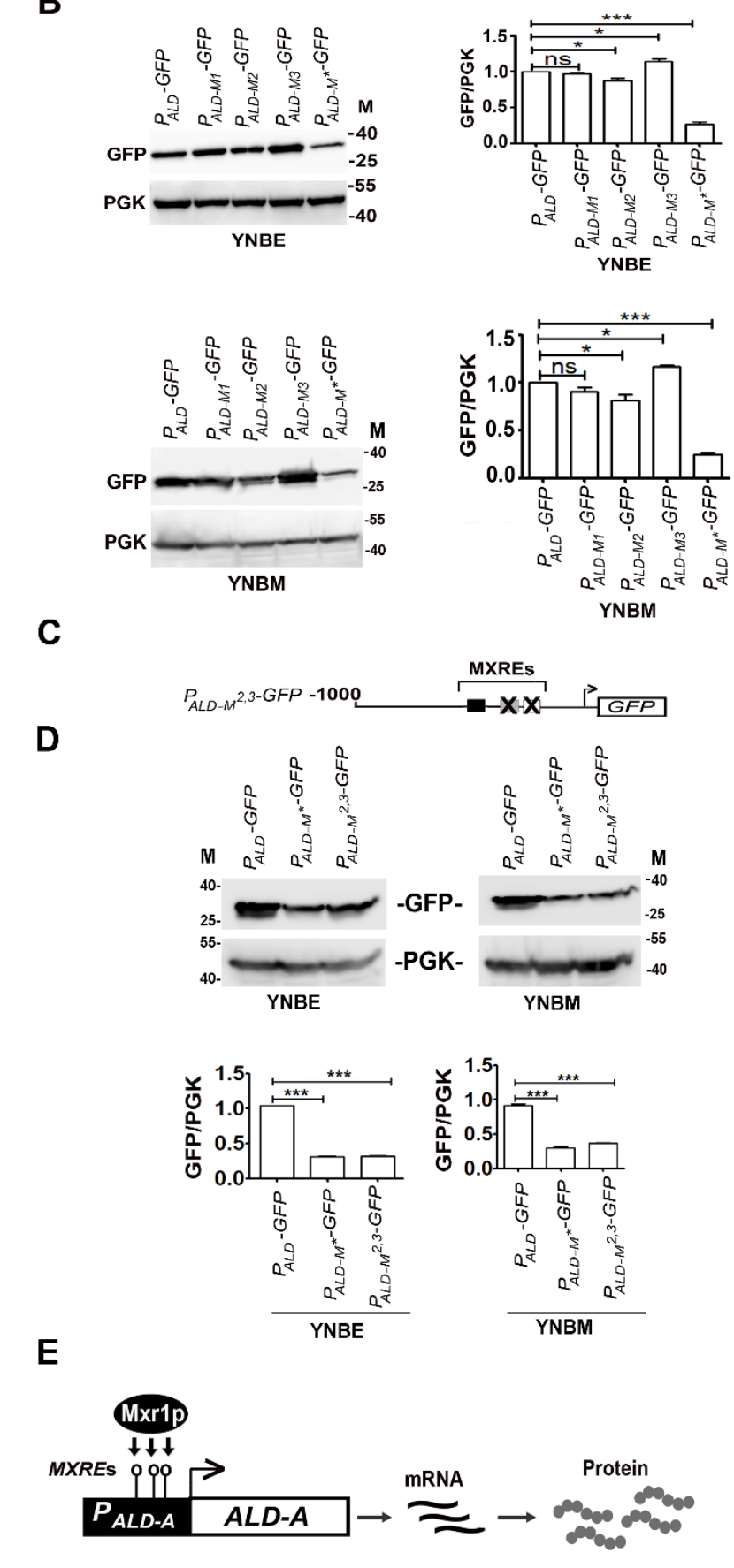

Fig. 4 
A

B

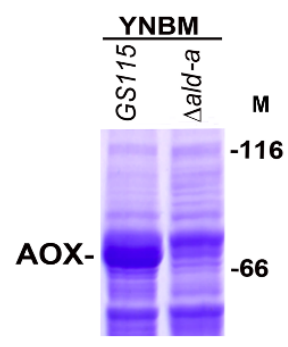

C

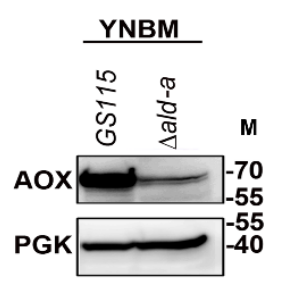

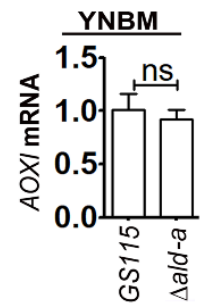

D

E

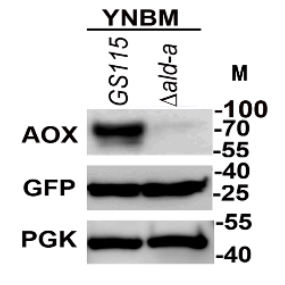

GS115-P AOX1 $_{1}-$ GFP

F
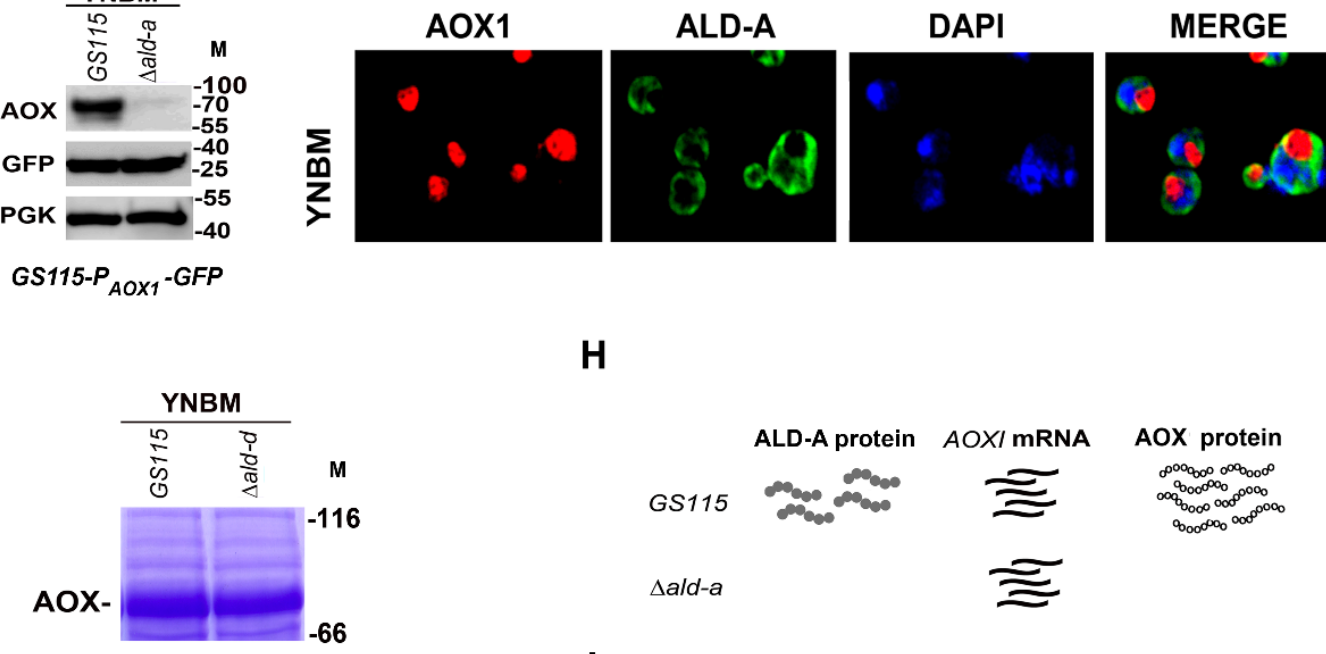

G

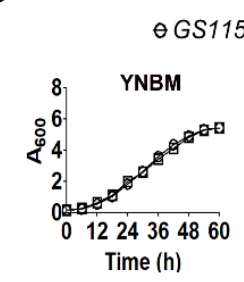

ㅂald-d

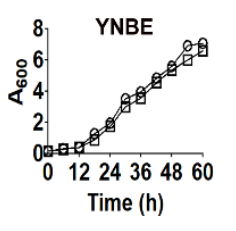

H

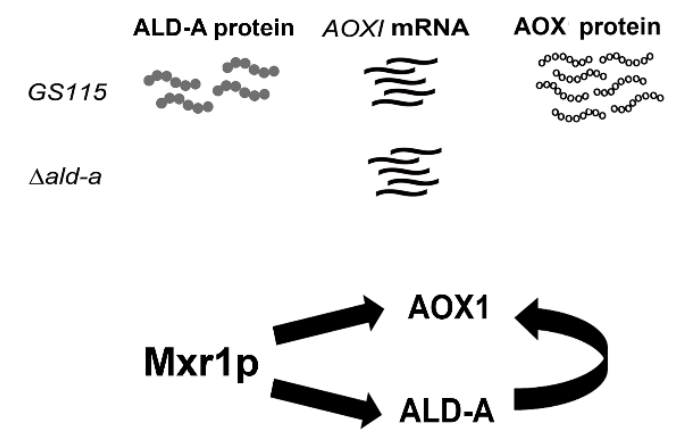

Fig. 5 


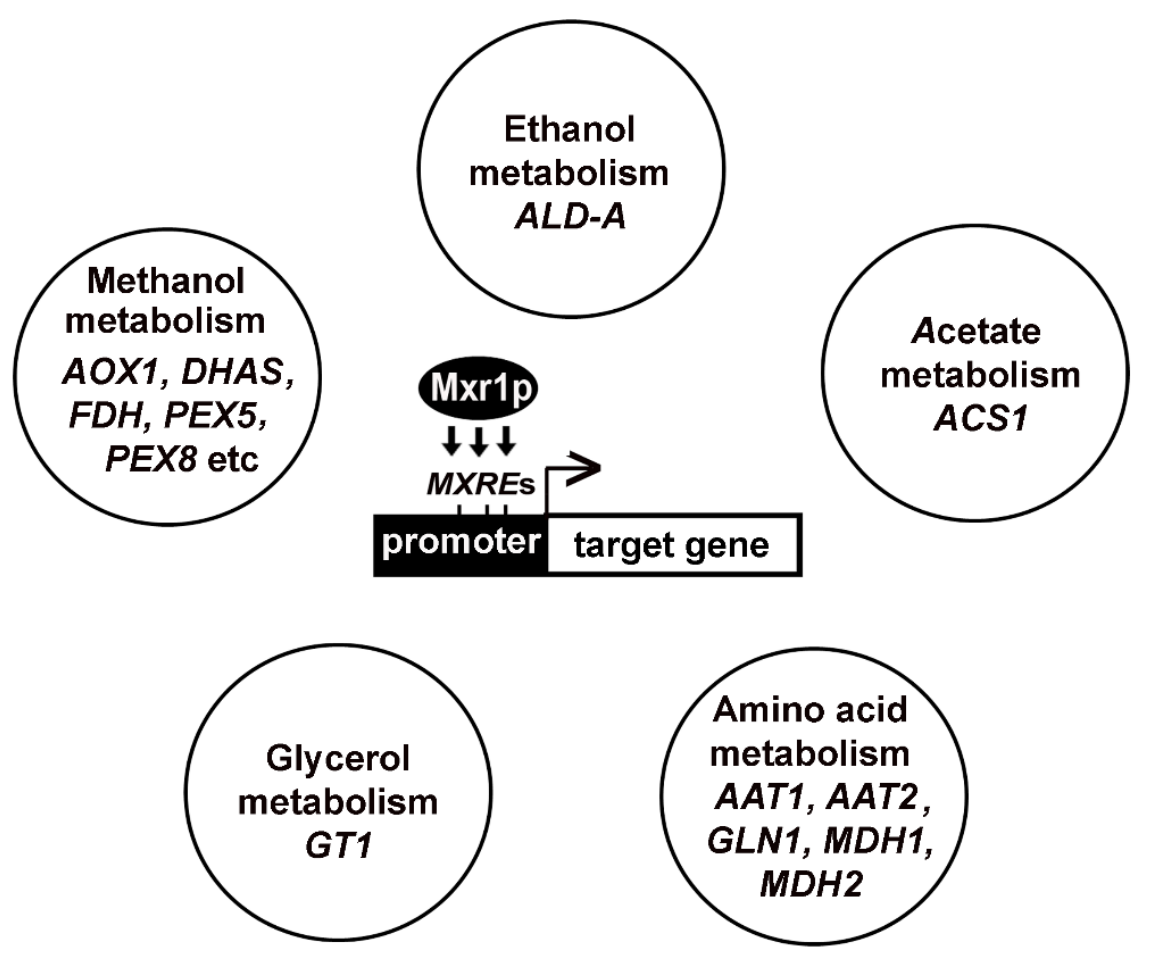

Fig. 6 